\title{
A new DFT based Channel Estimation Method for OFDM Systems over Multipath Channels
}

\author{
Jing Wang \\ Xi'an Institute of Optics and Precision Mechanics \\ Chinese Academy of Sciences \\ Xi'an, China, 15091892457 \\ wangjing2937@gmail.com
}

\author{
Yuehong Qiu \\ Xi'an Institute of Optics and Precision Mechanics \\ Chinese Academy of Sciences \\ Xi'an, China \\ yhqiu@opt.ac.cn
}

\begin{abstract}
Channel estimation is one of the most significant technologies in the orthogonal frequency division multiplexing (OFDM) system and its accuracy will influence the performance of the whole system directly. Among all kinds of channel estimation algorithms, the least square (LS) estimation and the linear minimum mean-squared error (LMMSE) estimation are the most classic ones. However, both the two estimator are not able to reach a compromise between accuracy and computational complexity. The discrete Fourier transform (DFT) -based channel estimation can get a better performance by a time domain processing. In this paper, we proposed an improved DFT based channel estimation which suppresses the noise by a new threshold setting method. The computer simulation results demonstrate that the proposed algorithm can reach accuracy close to the LMMSE algorithm and its computational complexity is much lower than the LMMSE estimator.
\end{abstract}

Keywords-orthogonal frequency division multiplexing;channel estimation;DFT;threshold

\section{INTRODUCTION}

Orthogonal Frequency Division Multiplexing (OFDM) technique has the advantage of high spectral efficiency and resistance to Multipath Fading. For this reason, it has received broad attention for the next generation mobile communication and wireless LAN area, and has been adopted as the key technology of physical layer for a variety of Protocols, such as LTE, IEEE802.11 and WiMAX. In order to achieve better performance, OFDM used to adopt coherent receiver, so it needs accurate channel estimation, which has a direct influence on the whole system performance. OFDM system channel estimation methods are basically divided into two classes: pilot-aided channel estimation and blind channel estimation. Blind channel estimation is rarely used for its high complexity and slow convergence [1]. However, pilot-aided channel estimation is used extensively for fast rate and good accuracy [2]. Pilot-aided channel estimation design is confronted mainly with three issues: Firstly, the choice of pilot. Based on different channel environment, there are many different manners to choose from, such as block pilot interpolation, comb pilot interpolation and scattered pilot interpolation. Secondly, the determination of channel estimation principals at pilot signals. Least Square (LS) Principal is easy for implementation, and does not need prior channel statistic information, but the result is badly affected by noise and the accuracy is poor. Algorithms

This work is supported by the nature scientific foundation of China, No.10978005/A030801 and No.61178033/F05070. based on Minimum Mean Squared Error (MMSE) Principal take advantage of channel statistic information, and can achieve ideal result, but have high complexity and are hard for real-time implementation [3]. Thus researchers have suggested many different algorithms to suppress noise for LS method and lower complexity for MMSE method [4]. Among these algorithms, one kind of algorithms based on DFT has received close attention for their good compromise between performance and complexity [5]. Thirdly, the interpolation algorithms to estimate channel response at data signals positions after pilot channel estimation has been achieved. This paper offers a deep investigation for the DFT-based channel estimation algorithms, and put forward an improved one. The improved algorithm has been run on MATLAB environment. The result has been compared with other algorithms, and shows that our algorithm achieves a better compromise between complexity and performance.

\section{OFDM SYSTEM BASEBAND MODEL}

An OFDM system with $\mathrm{N}$ subcarriers is shown in Fig.1

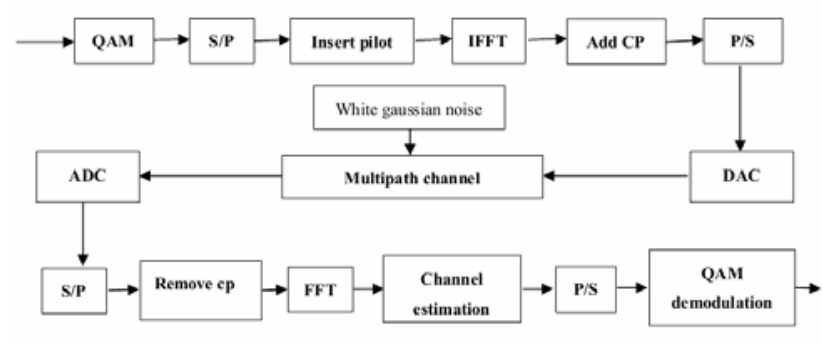

Figure 1. OFDM system baseband model

At the transmitter, the high rate input data are first mapped according to a modulation scheme (we used quadrature amplitude modulation ( QAM) ), then the serial to parallel conversion are performed to convert the high rate data to low rate parallel data flows. After pilot insertion, the data flows are modulated on the corresponding orthogonal subcarriers by IFFT. The cyclic prefix (CP) which contains a copy of the last part of the OFDM symbol is added to avoid the inter-symbol interference (ISI) and the inter-subcarrier interference (ICI). After passing through the multipath channel with additive Gaussian noise, the signals are recovered through a series of inverse processing at the receiver. 
We assumed that the number of the subcarriers is $\mathrm{N}$, the time domain channel impulse response ( CIR ) of the multipath fading channel can be represented as [3]

$$
h(n)=\sum_{l=0}^{L-1} \alpha_{l} \delta\left(n-\tau_{l}\right) \quad n=0,1, \ldots N-1
$$

where $\mathrm{L}$ is path number of the channel, $\alpha_{l}$ represents the complex gain of each path, $\tau_{l}$ is the delay of each path. We assumed that the channel is a slow fading channel which means it keeps unchanged during one OFDM symbol and the length of the CIR is shorter than that of the CP.

The channel frequency response (CFR) is expressed as

$$
H(k)=\sum_{l=0}^{L-1} \alpha_{l} e^{-j \frac{2 \pi}{N} k \tau_{l}} \quad k=0,1, \ldots N-1
$$

The frequency response on the kth subcarrier is $\mathrm{H}(\mathrm{k})$, and then the parallel Gaussian channels can be described in Fig.2 [5].

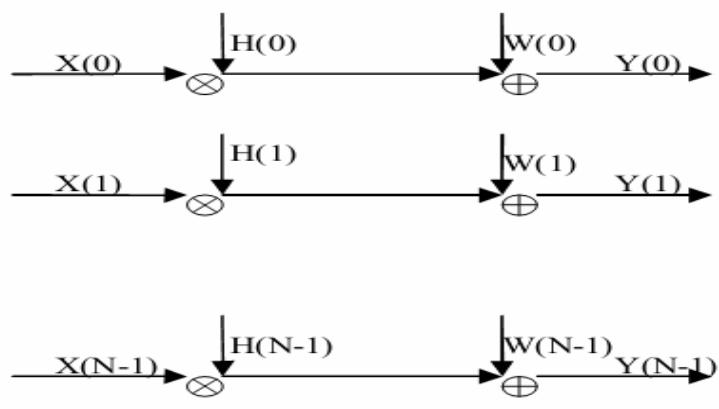

Figure 2. Parallel Gaussian channels

We can express the system by matrix as

$$
\mathrm{Y}=\mathrm{XH}+\mathrm{W}
$$

where $\mathrm{Y}$ is received vector of size $\mathrm{K}^{*} 1, \mathrm{X}$ is a diagonal matrix of size $\mathrm{N}^{*} \mathrm{~N}$ with its diagonal elements of transmitted signals, $\mathrm{H}$ represents the CFR vector and $\mathrm{W}$ is a vector of independent identically distributed Gaussian noise with a mean value of 0 and a variance of $\sigma^{2}$.

\section{ANALYSIS OF CLASSIC CHANNEL ESTIMATION ALGORITHMS}

In this section, we will introduce and analyze some classic channel estimators including the least square (LS) estimator, the linear least minimum mean-squared error (LMMSE) estimator, the low rank LMMSE (LRLMMSE) estimator and the DFT-based estimator.

LS estimator is simplest channel estimator which aims at minimize the square of the difference between the $\mathrm{Y}$ and $X \hat{H}_{L S}$ [3]

$$
\hat{H}_{L S}=\arg \min \left|Y-X \hat{H}_{L S}\right|
$$

LS estimation can be got by further deducing

$$
\hat{H}_{L S}=X^{-1} Y=H+\tilde{W}
$$

where $\tilde{W}=X^{-1} W$. Obviously, estimation of every coefficient needs only one multiplication, but its drawback is that it is vulnerable to noise.

The LMMSE algorithm improves the accuracy of estimation by the statistical information of the channel to minimize the mean-squared error [3]. It is an optimal linear filter to the LS estimation.

$$
\hat{H}_{L M M S E}=K_{X} \hat{H}_{L S}
$$

Minimize the MSE to obtain

$$
K_{X}=R_{H H}\left(R_{H H}+\sigma_{W}^{2}\left(X X^{H}\right)^{-1}\right)^{-1}
$$

where $R_{H H}$ represents the auto-correlation matrix of $\mathrm{H}$. For simplicity, we can replace $\left(X X^{H}\right)^{-1}$ with $E\left\{\left(X X^{H}\right)^{-1}\right\}$, then

$$
K=R_{H H}\left(R_{H H}+\frac{\beta}{S N R} I\right)^{-1}
$$

where SNR is the signal to noise ratio of the system, the value of $\beta$ is decided by the constellation (in the case of 16QAM, $\beta=17 / 9$ ). Due to the operation of inversing the matrix, its computational complexity is much larger than LS estimation. Estimation of every channel coefficient requires $(\mathrm{N}+1)$ multiplications. Moreover, the auto-correlation matrix of channel is often unknown. So the LMMSE algorithm can not be used in practical receiver.

In order to reduce the complexity of the LMMSE method, researchers proposed a simplified algorithm named the low rank linear minimum mean-squared error ( LRLMMSE ) method by an approximation of low ranks. In this method, we first implement a singular value decomposition of the autocorrelation matrix of the channel, and only retain the $p$ eigenvalue of the maximum value. It maps the LS vector to a subspace of $p$ dimensions to suppress the noise. LRLMMSE algorithm is a good compromise between the accuracy and complexity [6].

The DFT-based channel estimation has received close attention for its good performance and easy implementation. Owing to the fact that the length of the OFDM symbol is much longer than that of the CIR, we transform the CIR to the time domain for further processing

$$
\hat{h}_{l s}(n)=\operatorname{IDFT}_{N}\left\{\hat{H}_{L S}(k)\right\}=h(n)+\widetilde{w}(n)
$$

The noise can be suppressed by selecting the informative coefficients of CIR

$$
h_{D F T}(n)=\left\{\begin{array}{ll}
\hat{h}_{L S}(n) & n \in \text { significant } \\
0 & \text { others }
\end{array}\right. \text { channel samples }
$$

Then the CFR can be obtained by DFT

$$
\hat{H}_{D F T}(k)=D_{F T}\left\{\hat{h}_{D F T}(n)\right\} \quad 0 \leq k \leq N-1
$$

Owing to the fast Fourier transform, the complexity of DFT-based algorithm does not increase obviously. The 
traditional DFT- based algorithm set the points outside the CP to zero and consider all the samples inside it as informative ones. However, there is still some samples which contains only noise without any information of the channel inside the CP. Therefore, it is necessary to reduce the noise inside the CP [7]. In the next section, we put forward a new method to choose the informative coefficients based on a threshold.

\section{THE PROPOSED METHOD}

According to the analysis in section III, not all the channel coefficients within the length of CP are significant channel taps. In fact, most of them do not correspond to any path of the channel and contain only noises. Therefore, we can enhance the accuracy of estimation by selecting the most significant channel coefficients and setting all other samples to zero [8]. In this section, we proposed a new method to select the most significant coefficients based on a threshold. We only retain the coefficients whose absolute values are larger than the threshold:

$$
\hat{h}_{p}(n)=\left\{\begin{array}{cc}
\hat{h}_{l s}(n) & \left|\hat{h}_{l s}(n)\right|^{2}>\text { threshold } \\
0 & \text { others }
\end{array}\right.
$$
Fig.3

The block diagram of the proposed algorithm is showed in

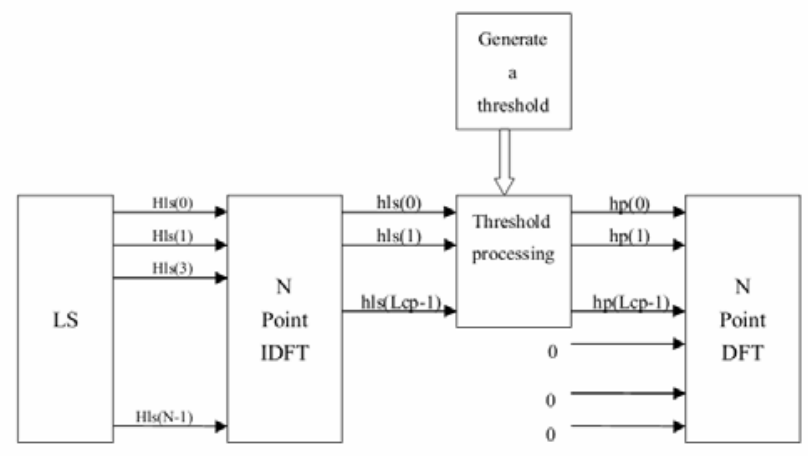

Figure 3. Block diagram of the proposed method

First, the LS estimation is implemented to obtain a rough estimation

$$
\hat{h}_{l s}(n)=\operatorname{IDFT}_{N}\left\{\hat{H}_{L S}(k)\right\}=h(n)+\widetilde{w}(n)
$$

The most critical step in our method is to determine the significant channel coefficients. Since length of the CP is much longer than that of the CIR, we consider all the samples longer than the length of $\mathrm{CP}$ as noises and set them to zero to obtain the

$$
\hat{h}_{t}(n)= \begin{cases}\hat{h}_{l s}(n) & 0 \leq n \leq L_{c p}-1 \\ 0 & \text { others }\end{cases}
$$

Now we have eliminated the noise outside the CP, the following task is to determine the threshold to suppress the noise inside it. The detailed step is elaborated as follows:

We calculate the total energy of the LS vector:

$$
E_{\text {totalls }}=\sum_{n=1}^{N-1}\left|\hat{h}_{l s}(n)\right|^{2}
$$

which is comprised of the two parts: the energy of the CIR and the energy of the noise:

$$
E_{\text {totalls }}=E_{\text {channells }}+E_{\text {noisels }}
$$

where $E_{\text {channells }}$ is the energy of the CIR, $E_{\text {noisels }}$ is the energy of noise.

Then we set the

$$
\frac{E_{\text {channells }}}{E_{\text {noisels }}}=r
$$

And the $r$ can be estimated by SNR

$$
\hat{r}=S N R
$$

According to equation (16), (17) and (18), we can get the energy of the CIR in the rough LS estimation

$$
\hat{E}_{\text {CHANNELLS }}=\frac{E_{\text {totalls }} \times S N R}{1+S N R}
$$

We consider the first $\mathrm{M}$ points with the maximum energy as the significant ones [9], so we sort the $\hat{h}_{t}(n)$ in a descended order according to the energy of every points to get $h_{\text {SORT }}(n)$.

Due to the energy of the CIR is $\hat{E}_{\text {CHANNELLS }}$, we have good reason to consider that the first M points in $h_{\text {SORT }}(n)$ are the significant channel coefficients if the sum of their energy equals $E_{\text {channells }}$.

So we in turn calculate the sum of energy of the largest $\mathrm{M}$ points in $h_{S O R T}(n)$

$$
E_{\text {sum }}(M)=\sum_{n=0}^{M-1}\left|h_{\text {sort }}(n)\right|^{2} \quad M=1,2, \ldots L_{c p}
$$

And when the $E_{\text {sum }}(M)$ exceeds the $E_{\text {channells }}$ for the first time, the above calculate stops and the threshold is set as thrershold $=h_{\text {sort }}(M)$

After threshold processing, we get the $\hat{h}_{p}(n)$. So the final frequency estimation can be obtained by FFT

$$
\hat{H}_{p}(k)=\operatorname{FFT}_{N}\left\{\hat{h}_{p}(n)\right\}
$$

\section{SIMULATIONS AND ANALYSIS}

In this section, we investigate the performance of the proposed method by computer simulation and compare it with some other typical algorithms. For a better comparison of the performance of the channel estimations, we assumed that there is no channel coding and synchronization of the system is perfect. The channel has 5 paths and the amplitude of each path 
varies independently with an exponential power delay profile. The other parameters of this simulation are showed in table I .

TABLE I .SIMULATION PARAMETERS

\begin{tabular}{|c|c|}
\hline Parameter & Value \\
\hline Number of subcarriers & 128 \\
\hline Modulation & 16QAM \\
\hline Interval between subcarriers & $7.8125 \mathrm{kHz}$ \\
\hline The length of CP & 16 \\
\hline Number of OFDM symbols & 100 \\
\hline
\end{tabular}

We investigated the mean square error (MSE) and the bit error rate (BER) of each method which are demonstrated in Fig. 4 and Fig.5.

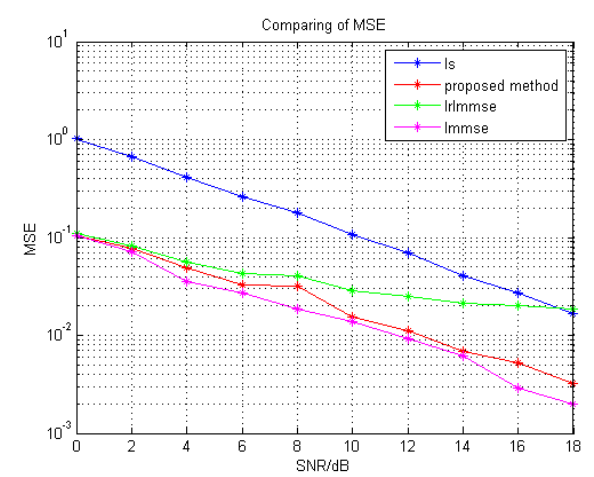

Figure 4. Comparing of MSE

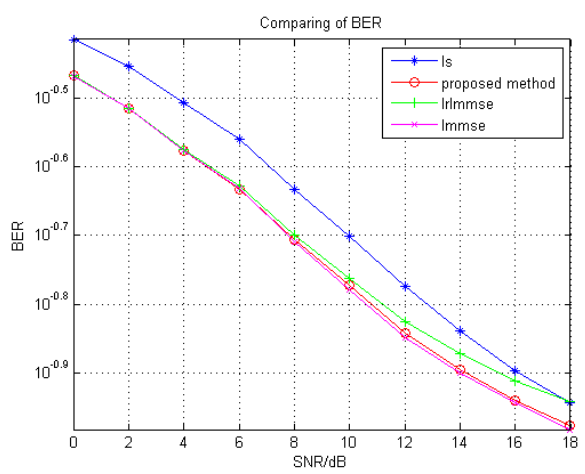

Figure 5. Comparing of BER

As is shown in Fig.4, the LS estimation got the worst MSE performance because of its vulnerability to noise and the LMMSE estimation performs best at the cost of high complexity. The other two curves are two compromising schemes and the proposed method outperforms the LRLMMSE method. Fig. 5 demonstrates the BER performance of the above four algorithms. We can see that it is similar with the MSE curves. The proposed method got an obvious improvement comparing to the LS and the LRLMMSE method and its BER performance got close to the best one.

As is analyzed in section III , the computational complexity of the LS and LMMSE estimator are 1 and $(\mathrm{N}+1)$ multiplications per coefficient respectively. The proposed method requires two Fourier transform which need $\log _{2} N$ multiplications, but the threshold processing does not need complex operation of matrix, so its computational complexity is low. The LRLMMSE estimator can also get a low complexity but its performance is poorer than our method.

\section{CONCLUSION}

This paper makes a study on channel estimation algorithms for OFDM system, and puts forward an improved DFT-based channel estimation algorithm to make a good compromise between accuracy and complexity. The new method chooses significant channel samples based on threshold setting, and suppresses noise inside the cyclic prefix. A comparison in broad aspects such as Bit Error Rate (BER), Mean Squared Error (MSE) and complexity has been made between our algorithm and several other classic algorithms. The simulation results show that our method has a better performance than LS and LRLMMSE algorithm and get close to the LMMSE algorithm in BER and MSE with a much lower complexity. Our method has made a good compromise between performance and complexity, and has good practical value.

\section{REFERENCES}

[1] S. A. Banani and R. G. Vaughan, "OFDM with iterative blind channel estimation,” IEEE Trans. Veh. Technol., vol. 59, pp. 4298-4308, Nov. 2010.

[2] Y. Li, "Simplified channel estimation for OFDM systems with multiple transmit antennas,” IEEE Trans. Wireless Communications, vol. 1, pp. 67-75, Jan. 2002.

[3] J.-J. van de Beek, O. Edfors, M. Sandell, S. K. Wilson, and P. O. Börjesson, "On channel estimation in OFDM systems," in Proc. IEEE 45th Vehicular Technology Conf., Chicago, IL, Jul. 1995, pp. 815-819.

[4] Anza Rani James, Revathy S Benjamin and Shilpa John, "Channel Estimation for OFDM Systems”, Proceedings of 2011 International Conference on Signal Processing, Communication, Computing and Networking Technologies (ICSCCN 2011)

[5] Ove Edfors , Magnus Sandell and Jan-Jpp Van De Beek, "Analysis of DFT-Based Channel Estimators for OFDM", Wireless Personal Communications 12: 55-70, 2000

[6] J. Lofgren, S. Mehmood, N. Khan, B. Masood, M. Awan and I. Khan, "Hardware implementation of an SVD based MIMO OFDM channel estimator”, in Proc. NORCHIP, 2009

[7] You-Seok Lee, Hyun-Chool Shin, and Hyoung-Nam Kim, “ Channel Estimation Based on a Time-Domain Threshold for OFDM Systems”,IEEE Trans. Broadcasting, vol. 55, NO. 3, Sep. 2009

[8] Y. Kang, K. Kim and H. Park, "Efficient DFT-based channel estimation for OFDM systems on multipath channels", IET Communications ,vol.1,n.2, pp. 197 - 202, April 2007.

[9] Najjar. L, "Sparse channels structured estimation in OFDM systems”,Proc. of VTC Spring 2011, Budapest, Hungary, May 2011 